\title{
Article \\ Clinical Outcomes of RTOG 9310 Protocol for Primary Central Nervous System Lymphoma: Single-Center Experience with 87 Patients
}

\author{
Jinuk Kim ${ }^{1}$, Tae Gyu Kim ${ }^{2}$, Hyoun Wook Lee ${ }^{3}$, Seok Hyun Kim ${ }^{4}$, Ji Eun Park ${ }^{5}$, Moonok Lee ${ }^{5}$ \\ and Young Zoon Kim ${ }^{1, *(D)}$
}

1 Division of Neuro Oncology, Department of Neurosurgery, Samsung Changwon Hospital, Sungkyunkwan University School of Medicine, Changwon 51353, Korea; jinukstar@gmail.com

2 Department of Radiation Oncology, Samsung Changwon Hospital, Sungkyunkwan University School of Medicine, Changwon 51353, Korea; ktg7757@skku.edu

3 Department of Pathology, Samsung Changwon Hospital, Sungkyunkwan University School of Medicine, Changwon 51353, Korea; sudowo@skku.edu

4 Division of Hematology and Medical Oncology, Department of Internal Medicine, Samsung Changwon Hospital, Sungkyunkwan University School of Medicine, Changwon 51353, Korea; tjrgus1@hanmail.net

5 Department of Anesthesiology and Pain Medicine, Samsung Changwon Hospital, Sungkyunkwan University School of Medicine, Changwon 51353, Korea; jnpark369@gmail.com (J.E.P.); lmo602@hanmail.net (M.L.)

* Correspondence: yzkim@skku.edu; Tel.: +82-55-233-5241; Fax: +82-55-233-6527

Citation: Kim, J.; Kim, T.G.; Lee, H.W.; Kim, S.H.; Park, J.E.; Lee, M.; Kim, Y.Z. Clinical Outcomes of RTOG 9310 Protocol for Primary Central Nervous System Lymphoma: Single-Center Experience with 87 Patients. Curr. Oncol. 2021, 28, 4655-4672. https://doi.org/10.3390/ curroncol28060393

Received: 1 November 2021 Accepted: 10 November 2021 Published: 12 November 2021

Publisher's Note: MDPI stays neutral with regard to jurisdictional claims in published maps and institutional affiliations.

Copyright: (c) 2021 by the authors. Licensee MDPI, Basel, Switzerland. This article is an open access article distributed under the terms and conditions of the Creative Commons Attribution (CC BY) license (https:// creativecommons.org/licenses/by/ $4.0 /)$.
Abstract: The Radiation Therapy Oncology Group (RTOG) 9310 protocol clinical trial established high-dose methotrexate (HDMTX) as the standard for primary central nervous system lymphoma (PCNSL). We aimed to investigate the RTOG 9310 protocol's PCNSL outcomes by examining progressionfree survival (PFS) and overall survival (OS) rates and determining the influential factors. Between 2007 and 2020, 87 patients were histopathologically diagnosed with PCNSL and treated with the RTOG 9310 protocol. All received HDMTX $2.5 \mathrm{~g} / \mathrm{m}^{2}$ and vincristine $1.4 \mathrm{mg} / \mathrm{m}^{2} /$ day for 1 day during weeks $1,3,5,7$, and 9 , and procarbazine $100 \mathrm{mg} / \mathrm{m}^{2} /$ day for 1 day during weeks 1,5 , and 9 . Dexamethasone was administered on a standard tapering schedule from the first week to the sixth week. Whole brain radiotherapy (WBRT), consisting of $45 \mathrm{~Gy}$ for patients with less than a complete response to the chemotherapy or 36 Gy for complete responders, was started 1 week after the last dose of chemotherapy was administered. Within three weeks of the completion of WBRT, patients received two courses of cytarabine, which were separated by 3-4 weeks. Clinical, radiological, and histopathological characteristics were retrospectively reviewed. All patients completed five HDMTX cycles and a mean follow-up of 60.2 (range, 6-150) months. Twenty-eight (32.2\%) patients experienced recurrence during follow-up. The mean time to recurrence was 21.8 months, while the mean PFS was 104.3 (95\% confidence interval (CI), 90.6-118.0) months. Eleven (12.6\%) patients died; the mean OS was 132.1 (95\% CI, 122.2-141.9) months. The 3- and 5-year survival rates were $92.0 \%$ and $87.4 \%$, respectively. One patient experienced acute renal failure, while the remainder tolerated any cytotoxic side effects. On multivariate analysis, the Eastern Cooperative Oncology Group performance score $\leq$ 2; the International Extranodal Lymphoma Study Group low-risk status; XBP-1, p53, and c-Myc negativity; homogenous enhancement; gross total resection, independently correlated with long PFS and OS. The RTOG 9310 protocol is effective for PCNSL and features good outcomes.

Keywords: adverse effect; chemotherapy; methotrexate; primary CNS lymphoma; prognosis

\section{Introduction}

Primary central nervous system lymphoma (PCNSL) is a highly aggressive extranodal subtype of non-Hodgkin lymphoma that is usually confined to the brain, eyes, leptomeninges, or spinal cord in the absence of systemic lymphoma. More than $95 \%$ of 
PCNSL cases have a histology comparable to that of diffuse large B-cell lymphoma (DLBCL) [1]. PCNSL are rare tumors that account for up to $1 \%$ of all lymphomas, $4-6 \%$ of all extranodal lymphomas, and approximately $3 \%$ of all primary brain tumors worldwide, including Korea [2]. Immunocompromised individuals such as those with human immunodeficiency virus (HIV) and Epstein-Barr virus (EBV) infections are considered most at risk of developing PCNSL; however, its incidence is increasing in immunocompetent populations and now represents the vast majority of patients [3-5]. PCNSL typically follows an aggressive course and, despite treatment advances, remains associated with very high mortality rates [1].

Although PCNSL is a highly malignant tumor with a poor prognosis in untreated patients in contrast to most malignant brain tumors, it is sensitive to corticosteroids, chemotherapy, and radiotherapy, which can achieve complete remission (CR) and extended long-term survival. For systemic non-Hodgkin lymphoma, the cyclophosphamide, doxorubicin, vincristine, and prednisolone regimen is routinely used and induces responses with relatively good outcomes. However, this regimen is not recommended for PCNSL because it offers no survival advantage over radiotherapy alone [6-8]. The apparent inability of these chemotherapeutic regimens to cross the brain-blood barrier and eradicate microscopic disease is a major challenge. To date, intravenous high-dose methotrexate (HDMTX) is considered the most important and beneficial single drug for PCNSL based on convergent results from many prospective and retrospective studies $[1,9,10]$. Although HDMTX is the backbone for the treatment of newly diagnosed PCNSL, neither a concrete combination regimen nor an effective dose has been conclusively established. Controversy persists about which type of consolidation regimen may be most beneficial after HDMTX-based induction therapy.

Whole brain radiotherapy (WBRT) was used to treat newly diagnosed PCNSL until the early 1980s, and the introduction of HDMTX plus WBRT improved clinical outcomes with a median overall survival (OS) of 30-60 months and 5-year survival rates of 30-50\% [11]. Rituximab, a monoclonal antibody directed against the B-cell surface antigen CD20, recently, dramatically improved the response and clinical outcomes of DLBCL and was incorporated into first-line PCNSL treatment regimens. The therapeutic outcome has improved substantially in the past two decades as a result of better curative treatment strategies. However, the treatment of this disease remains challenging because remission is frequently of short duration and recurrence is not prevented.

The Radiation Therapy Oncology Group (RTOG) 9310 protocol of combination chemotherapy composed of HDMTX, procarbazine, and vincristine followed by radiotherapy for PCNSL is a traditional regimen with good therapeutic outcomes [12,13]. Their report of the first multicenter trial demonstrated improved survival with the combination of chemotherapy and radiotherapy compared with previous reports of radiotherapy alone. They showed that an HDMTX-based regimen produced a high response rate before radiotherapy was administered and HDMTX plus cranial irradiation effectively treated PCNSL. As neurotoxicity was considered a delayed risk of the RTOG 9310 protocol, the dose of radiotherapy was reduced from $45 \mathrm{~Gy} / 25$ fractionation to $36 \mathrm{~Gy} / 30$ fractionation in a secondary analysis [13]. This analysis showed that progression-free survival (PFS) and OS rates were not significantly affected despite the hyperfractionated radiotherapy schedule representing a $25 \%$ reduction in biologically effective tumor dose [13].

After the report of improved clinical results from the RTOG 9310 protocol for PCNSL patients was published in 2005, we started to treat newly diagnosed PCNSL patients using this protocol at our institute. In the present study, we primarily investigated the PFS and OS of PCNSL patients treated with the RTOG 9310 protocol. We also examined the predictive factors associated with PFS and OS to be validated with previously known prognostic factors in the literature. This analysis included pathological markers that were not addressed in the previous RTOG study for predicting clinical outcomes. Several tips for the application of the RTOG 9310 protocol from our experience are discussed to reduce the adverse effects and improve the treatment success. 


\section{Materials and Methods}

\subsection{Patient Enrolment}

Newly diagnosed immunocompetent patients with PCNSL who were treated at our institute between March 2007 and August 2020 were evaluated for study participation. Inclusion and exclusion criteria were applied as in the RTOG 9310 protocol [12]. The inclusion criteria were as follows: histologic proof of non-Hodgkin lymphoma by brain biopsy; histological diagnosis received within the previous 4 weeks; absolute neutrophil count $(\mathrm{ANC}) \geq 2000$ cells $/ \mathrm{mm}^{3}$; platelets $\geq 100,000$ cells $/ \mathrm{mm}^{3}$; total bilirubin $\leq 2.0 \mathrm{mg}$; serum glutamic oxaloacetic transaminase (SGOT) $\leq 2$ times the upper limit of normal; creatinine clearance $\geq 50 \mathrm{cc} / \mathrm{min} / 1.73 \mathrm{~m}^{2}$; normal serum electrolytes; a life expectancy of at least 8 weeks; Eastern Cooperative Oncology Group (ECOG) performance score $\leq 3$; HIV-1 negative serology. The exclusion criteria were as follows: prior cranial irradiation; concurrent malignancies; pre-existing immunodeficiency such as renal transplantation; prior treatment with chemotherapy; ECOG performance score $\geq 4 ; \mathrm{ANC}<2000 \mathrm{cells} / \mathrm{mm}^{3}$; platelets $<100,000$ cells $/ \mathrm{mm}^{3}$; total bilirubin $>2.0 \mathrm{mg}$; SGOT $>2$ times the upper limit of normal; creatinine clearance $<50 \mathrm{cc} / \mathrm{min} / 1.73 \mathrm{~m}^{2}$; abnormal serum electrolytes; currently pregnant or nursing. Patients who were excluded from this protocol were scheduled to undergo radiotherapy alone or supportive care.

To exclude evidence of systemic lymphoma, a negative staging evaluation using chest, abdominal, and pelvic computed tomography and bone marrow biopsy was required. All patients underwent cranial neuroimaging at the time of diagnosis, preferably using magnetic resonance image (MRI). All patients underwent lumbar puncture and complete ophthalmologic evaluation, including a slit-lamp examination. The ECOG performance status definition was used [14]. Before chemotherapy, a laboratory diagnosis of EBV infection was made on a single serum sample using a standard immunofluorescence test for antibodies to EBV-associated antigens, simultaneously; immunoglobulin $\mathrm{M}$ and $\mathrm{G}$ to the viral capsid antigen, the early antigen, and the EBV nuclear antigen.

\subsection{Treatment Protocol}

The protocol was performed as previously reported by the RTOG [12]. Chemotherapy was administered for five cycles over a 10-week period (Supplementary Table S1). Each cycle consisted of methotrexate $2.5 \mathrm{~g} / \mathrm{m}^{2}$ infused over $2-3 \mathrm{~h}$ and vincristine $1.4 \mathrm{mg} / \mathrm{m}^{2}$ with a cap of $2.8 \mathrm{mg}$. Methotrexate was followed by vigorous hydration at a rate of $1500-1800 \mathrm{~mL} / \mathrm{m}^{2}$ for the first $24 \mathrm{~h}$, followed by a rate of $2000 \mathrm{~mL} / \mathrm{m}^{2}$ for the subsequent $48 \mathrm{~h}$. Urine alkalinization was accomplished with intravenous bicarbonate, and rescue leucovorin $20 \mathrm{mg}$ orally every $6 \mathrm{~h}$ for 12 doses was initiated $24 \mathrm{~h}$ after methotrexate administration. In addition to methotrexate and vincristine, procarbazine $100 \mathrm{mg} / \mathrm{m}^{2} /$ day for 7 days was administered during cycles 1,3, and 5. All patients with positive CSF findings underwent Ommaya reservoir placement and the administration of methotrexate $12 \mathrm{mg}$ for five cycles the week after each dose of intravenous methotrexate. Intra-Ommaya methotrexate was followed by oral leucovorin $10 \mathrm{mg}$ every $6 \mathrm{~h}$ for eight doses immediately after each dose of intrathecal MTX administration. Dexamethasone was administered on a standard tapering schedule of $16 \mathrm{mg} /$ day for the first week, $12 \mathrm{mg} /$ day for week $2,8 \mathrm{mg} /$ day for week 3, $6 \mathrm{mg}$ /day for week 4, $4 \mathrm{mg} /$ day for week 5, and $2 \mathrm{mg} /$ day for week 6; however, the dose could be adjusted according to the patient's neurological condition. All patients received trimethoprim/sulfamethoxazole prophylaxis for Pneumocystis carinii pneumonia.

WBRT was planned for a total dose of $45 \mathrm{~Gy}$ in 1.80-Gy fractions. If ocular lymphoma was present, both eyes were included in the radiotherapy field to a total dose of $36 \mathrm{~Gy}$ in 20 fractions. Approximately halfway through the study, there was growing evidence from the RTOG report that long-term survivors of combined methotrexate-based chemotherapy and cranial irradiation developed permanent severe neurotoxicity [12]. This is a particularly significant issue for older patients. Our institute's protocol was then modified so that those patients who achieved a CR at the end of 10 weeks of chemotherapy would receive a 
reduced dose of WBRT course for a total dose of 36 Gy given in 1.8-Gy fractions for 20 days, which was started 1 week after the last dose of chemotherapy was administered [12].

At the completion of cranial irradiation, all patients were scheduled to receive two courses of high-dose cytarabine within three weeks of the completion of WBRT according to the original RTOG protocol. Each course consisted of two doses separated by $24 \mathrm{~h}$ of cytarabine $3 \mathrm{~g} / \mathrm{m}^{2} /$ day infused over $3 \mathrm{~h}$ (Supplementary Table S1).

When the disease recurred or progressed, repeated HDMTX-based combined chemotherapy regimen was used based on the initial RTOG 9310 protocol, and was considered primarily without brain irradiation.

\subsection{Clinical Data Collection}

Epidemiological characteristics (including sex, age at the time of the PCNSL diagnosis, and performance status), type of primary treatment for PCNSL, type of salvage treatment for recurrent and progressive disease (PD), duration of follow-up, and time of death were retrospectively reviewed from the medical records of each patient. For prognostic assessment, additional factors, such as the serum lactate dehydrogenase (LDH) level, protein concentration in the cerebrospinal fluid (CSF), and deep brain involvement, were evaluated using the literature criteria provided by International Extranodal Lymphoma Study Group (IELSG) [15]. All patients were treated with the same protocol of high-dose methotrexate-based combination chemotherapy followed by adjuvant WBRT.

The radiological characteristics of the brain lesions were evaluated using MRI at the time of the initial diagnosis of PCNSL. The number was classified as unifocal or multifocal based on whether the mass was enhanced with gadolinium on T1-weighted MRI. The basal ganglia, corpus callosum, brain stem, and cerebellum were defined as deep brain structures. Peritumoral edema was categorized as $<$ or $\geq 2 \mathrm{~cm}$ from the brain tumor as assessed by T2-weighted MRI. In cases of multiple brain lesions, regardless of their number, different patterns of enhancement on MRI were considered heterogeneous enhancement. The radiological evaluation was performed by two different neuroradiologists (Y.M. Kim, Samsung Changwon Hospital, Changwon, Korea and M.O. Sunwoo, Samsung Changwon Hospital, Changwon, Korea) who were blinded to the clinical and pathological parameters.

In terms of histopathological characteristics, a routine analysis of the diagnostic markers was performed at the time of the initial diagnosis, such as pathological diagnosis according to the 2016 World Health Organization classification [16]; cell type; EBV in situ hybridization; Ki67 index; BCL2 or BCL6, p53, MUM1, and c-Myc immunoreactivity. According to the literature, the cutoff value for meaningful immunoreactivity was defined as "positive" immunohistochemical staining of at least $40 \%$ of the tumor cells for c-Myc and 30\% for BCL2 or BCL6 [17]. These features were obtained from pathological reports without additional immunohistochemical staining.

\subsection{Follow-Up}

Treatment response was assessed by contrast-enhanced brain MRI performed no more than 7 days before the commencement of chemotherapy, after the second and fifth HDMTX chemotherapy cycles, and 4 weeks after the termination of chemotherapy. The National Cancer Institute standardized response criteria regarding changes in enhanced lesion size on T1-weighted MR images were used to define treatment response [18]. In brief, a CR was defined as the complete disappearance of all evidence of lymphoma, partial response (PR) as a $\geq 50 \%$ decrease in tumor size, PD as a $\geq 25 \%$ increase in tumor size or the appearance of any new lesion, and stable disease (SD) as none of the above. Progression was defined as a new lesion with enhancement on MRI or an increase in tumor size by $25 \%$ or more. The time to progression and PFS were calculated from the diagnosis of PCNSL.

Repeat neuroimaging was required after the completion of neoadjuvant chemotherapy and radiotherapy, every 2 months for 1 year, every 4 months for the second year, and every 6 months thereafter. A comprehensive review of the chemotherapy records was performed to assess protocol adherence, and a radiologic review was performed by two 
neuroradiologists to assess the response to neoadjuvant chemotherapy as mentioned above. All adverse effects were recorded during the follow-up period according to the Common Terminology Criteria for Adverse Events provided by the National Cancer Institute [19].

\subsection{Survival and Statistical Analyses}

The medical records of the clinical history and radiographic reports of all study subjects were analyzed. The date of death was confirmed and recorded. OS was defined as the time from the date of the histological diagnosis of PCNSL until death. The date of biopsy or surgical resection of the brain lesion was recorded as the date of diagnosis.

The statistical analyses were performed using SPSS ver. 20.0 (IBM Corp., Armonk, NY, USA). Differences between subgroups were analyzed with Student's t-test for normally distributed continuous values, the Mann-Whitney U test for non-normally distributed continuous values, and chi-squared tests for categorical variables. OS and PFS were calculated using the Kaplan-Meier method. Comparisons among groups were performed using the log-rank test. Variables that were significantly associated with a longer OS and PFS on the univariate analyses were examined using multivariate analysis. Several additional variables associated with OS and PFS in the literature and of interest to the investigators were also included in the multivariate analysis. In this analysis, the Cox proportional hazards regression model was used to assess the independent effects of specific factors on OS and PFS and define the hazard ratios of the significant covariates. Differences were considered statistically significant at two-sided values of $p<0.05$.

As there is no universal cutoff value for the several clinical factors that predict OS of PCNSL, receiver operating characteristic curve analysis and a sensitivity-specificity analysis were used to define the cutoff value for the pathological biomarkers BCL2, BCL6, p53, MUM1, and c-Myc as predictive factors for OS of PCNSL. Through the sensitivityspecificity analysis, the cutoff value (the point at which sensitivity and specificity intersect) was determined for each value as correlated with survival [20].

\section{Results}

\subsection{Patient and Tumor Characteristics}

Among 100 patients who were newly diagnosed with PCNSL through brain biopsy or resection between March 2007 and August 2020, eight patients refused chemotherapy and underwent radiotherapy alone. Another five patients underwent supportive care alone instead of the HDMTX-based combination chemotherapy because they did not meet the eligibility criteria, for instance, three had an ECOG performance score of 4 and two had creatinine clearance $<50 \mathrm{cc} / \mathrm{min} / 1.73 \mathrm{~m}^{2}$. Ultimately, 87 patients were enrolled in the study after 13 patients were excluded. The study population included 45 men (51.7\%) and 42 women $(48.3 \%)$. The mean age of these patients at the time of the PCNSL diagnosis was 57.6 years (range, 32.4-81.2 years). Six patients $(6.9 \%)$ had ocular tumor involvement. Serum lactate dehydrogenase (LDH) levels were elevated in 32 patients $(36.8 \%)$, while cerebrospinal fluid (CSF) protein concentrations were elevated in 54 patients $(62.1 \%)$. Sixtytwo patients (71.3\%) exhibited an independent performance status in daily activity with an Eastern Cooperative Oncology Group (ECOG) performance score of 0-1, while 25 patients $(28.7 \%)$ exhibited a score of $2-3$. In terms of risk evaluation for prognosis, 28 patients $(32.2 \%)$ were categorized as low risk (International Extranodal Lymphoma Study Group (IELSG) score of 0-1), 24 (39.1\%) as intermediate risk (IELSG score of 2-3), and 25 (28.7\%) as high risk (IELSG score of 4-5) (Table 1). There was a significant difference in recurrence rate according to the ECOG performance score $(0-1$ versus $2-3 ; p=0.037)$ and the risk group $(p=0.048)$ (Table 1$)$. 
Table 1. Clinical features of 87 patients with primary central nervous system lymphoma.

\begin{tabular}{|c|c|c|c|c|}
\hline Features & $\begin{array}{c}\text { Total } \\
(n=87)\end{array}$ & $\begin{array}{l}\text { Recurrence }(+) \\
\quad(n=28)\end{array}$ & $\begin{array}{c}\text { Recurrence }(-) \\
\quad(n=59)\end{array}$ & $p$ Value \\
\hline Age & & & & 0.068 \\
\hline$<60$ years & $49(56.3 \%)$ & $10(20.4 \%)$ & $39(79.6 \%)$ & \\
\hline$\geq 60$ years & $38(43.7 \%)$ & $18(47.7 \%)$ & $20(52.6 \%)$ & \\
\hline Gender & & & & 0.923 \\
\hline Male & $45(51.7 \%)$ & $14(31.1 \%)$ & $31(68.9 \%)$ & \\
\hline Female & $42(48.3 \%)$ & $14(33.3 \%)$ & $28(66.7 \%)$ & \\
\hline Ocular involvement & & & & 0.917 \\
\hline Yes & $6(6.9 \%)$ & $2(33.3 \%)$ & $4(66.7 \%)$ & \\
\hline No & $81(93.1 \%)$ & $26(32.1 \%)$ & $55(67.9 \%)$ & \\
\hline Elevated serum LDH & & & & 0.132 \\
\hline Yes & $32(36.8 \%)$ & $13(40.6 \%)$ & $19(59.4 \%)$ & \\
\hline Mo & $55(63.2 \%)$ & $15(27.3 \%)$ & $40(72.7 \%)$ & \\
\hline Elevated protein in CSF & & & & 0.426 \\
\hline Yes & $54(62.1 \%)$ & $19(35.2 \%)$ & $35(64.8 \%)$ & \\
\hline No & $33(37.9 \%)$ & $9(27.3 \%)$ & $24(72.7 \%)$ & \\
\hline ECOG performance score & & & & 0.037 \\
\hline $0-1$ & $62(71.3 \%)$ & $15(24.2 \%)$ & $47(75.8 \%)$ & \\
\hline $2-3$ & $25(28.7 \%)$ & $13(52.0 \%)$ & $12(48.0 \%)$ & \\
\hline Risk of IELSG & & & & 0.048 \\
\hline Low $(0-1)$ & $28(32.2 \%)$ & $2(7.1 \%)$ & $26(92.9 \%)$ & \\
\hline Intermediate (2-3) & $34(39.1 \%)$ & $13(38.2 \%)$ & $21(61.8 \%)$ & \\
\hline High $(4-5)$ & $25(28.7 \%)$ & $13(52.0 \%)$ & $12(48.0 \%)$ & \\
\hline Adjuvant cytarabine treatment & & & & 0.602 \\
\hline Yes & $19(21.8 \%)$ & $7(36.8 \%)$ & $12(63.2 \%)$ & \\
\hline No & $68(78.2 \%)$ & $21(30.8 \%)$ & $47(69.2 \%)$ & \\
\hline
\end{tabular}

Abbreviations: CSF, cerebrospinal fluid; ECOG, Eastern Cooperative Oncology Group; IELSG, International Extranodal Lymphoma Study Group; LDH, lactate dehydrogenase.

A radiological analysis showed 57 (65.5\%) unifocal lesions, 38 (43.7\%) cases of deep brain involvement, and five (5.7) cases of CSF seeding. The maximal tumor size was $\geq 3 \mathrm{~cm}$ in $40(46.0 \%)$ patients and peritumoral edema was $\geq 2 \mathrm{~cm}$ in $33(37.9 \%)$ patients. Homogeneous enhancement was observed in $19(21.8 \%)$ patients versus heterogeneous enhancement in $68(78.2 \%)$ patients. Thirty-one (35.6\%) patients underwent gross total resection, while the other $56(64.4 \%)$ underwent partial resection or brain biopsy (Table 2). There was a significant difference in the recurrence rate according to the enhancement pattern (homogenous versus heterogeneous; $p=0.042$ ) and surgical extent (gross total versus partial resection/biopsy; $p=0.036$ ) (Table 2).

In terms of histopathological features, there were $74(85.1 \%)$ cases of DLBCL and 32 (36.8\%) cases of germinal center B-cell-like lymphoma. The cutoff values of each pathological biomarker for determining immunohistochemical positivity and negativity were as follows: $30 \%$ for BCL2 (area under the curve (AUC), 0.688; sensitivity, $72.3 \%$; specificity, $65.4 \%$ ), $40 \%$ for BCL6 (AUC, 0.702 ; sensitivity, $74.1 \%$; specificity, $66.8 \%$ ), $15 \%$ for p53 (AUC, 0.693; sensitivity, $64.8 \%$; specificity, $71.5 \%$ ), 35\% at MUM1 (AUC, 0.726 ; sensitivity, $72.6 \%$; specificity, $74.2 \%$ ), $60 \%$ at c-Myc (AUC, 0.775 ; sensitivity, $79.6 \%$; specificity, $75.3 \%$ ), and $50 \%$ at Ki67 (AUC, 0.636; sensitivity, 64.9\%; specificity, 67.3\%). Immunohistochemical positivity for BCL2 was found in $66(75.9 \%)$ patients, BCL6 in 69 (79.3\%) patients, p53 in $38(43.7 \%)$ patients, MUM1 in 50 (57.5\%) patients, and c-Myc in 41 (47.1\%) patients. EBV infection was detected by in situ hybridization analysis in $19(21.8 \%)$ patients, while the Ki67 proliferative index was $\geq 50 \%$ in $56(64.4 \%)$ patients (Table 3$)$. There was a significant difference in recurrence rate according to 553 immunoreactivity (positive versus negative; $p=0.040$ ), MUM1 (positive versus negative; $p=0.009$ ), and c-Myc (positive versus negative; $p=0.027$ ) (Table 3). 
Table 2. Radiologic features of 87 patients with primary central nervous system lymphoma.

\begin{tabular}{|c|c|c|c|c|}
\hline Features & $\begin{array}{c}\text { Total } \\
(n=87)\end{array}$ & $\begin{array}{l}\text { Recurrence }(+) \\
\quad(n=28)\end{array}$ & $\begin{array}{c}\text { Recurrence }(-) \\
\quad(n=59)\end{array}$ & $p$ Value \\
\hline Patterns & & & & 0.674 \\
\hline Unifocal & $57(65.5 \%)$ & $17(29.8 \%)$ & $40(70.2 \%)$ & \\
\hline Multifocal & $30(34.5 \%)$ & $11(36.7 \%)$ & $19(63.3 \%)$ & \\
\hline Deep brain involvement & & & & 0.392 \\
\hline Yes & $38(43.7 \%)$ & $14(36.8 \%)$ & $24(63.2 \%)$ & \\
\hline No & $49(56.3 \%)$ & $14(28.6 \%)$ & $35(71.4 \%)$ & \\
\hline CSF seeding & & & & 0.882 \\
\hline Yes & $5(5.7 \%)$ & $2(40.0 \%)$ & $3(60.0 \%)$ & \\
\hline No & $82(94.3 \%)$ & $26(31.7 \%)$ & $56(68.3 \%)$ & \\
\hline Maximal size of tumor & & & & 0.071 \\
\hline$\geq 3 \mathrm{~cm}$ & $40(46.0 \%)$ & $18(45.0 \%)$ & $22(55.5 \%)$ & \\
\hline$<3 \mathrm{~cm}$ & $47(54.0 \%)$ & $10(21.3 \%)$ & $37(78.7 \%)$ & \\
\hline Peritumoral edema & & & & 0.338 \\
\hline$\geq 2 \mathrm{~cm}$ & $33(37.9 \%)$ & $13(39.4 \%)$ & $20(60.6 \%)$ & \\
\hline$<2 \mathrm{~cm}$ & $54(62.1 \%)$ & $15(27.8 \%)$ & $39(72.2 \%)$ & \\
\hline Enhancement patterns & & & & 0.042 \\
\hline Homogeneous & $19(21.8 \%)$ & $3(15.8 \%)$ & $16(84.2 \%)$ & \\
\hline Heterogeneous & $68(78.2 \%)$ & $25(36.8 \%)$ & $43(63.2 \%)$ & \\
\hline Surgical extent & & & & 0.036 \\
\hline Gross total resection & $31(35.6 \%)$ & $6(19.4 \%)$ & $25(80.6 \%)$ & \\
\hline Biopsy and partial resection & $56(64.4 \%)$ & $22(39.3 \%)$ & $34(60.7 \%)$ & \\
\hline
\end{tabular}

Table 3. Histopathological features of 87 patients with primary central nervous system lymphoma.

\begin{tabular}{|c|c|c|c|c|}
\hline Features & $\begin{array}{c}\text { Total } \\
(n=87)\end{array}$ & $\begin{array}{l}\text { Recurrence }(+) \\
\quad(n=28)\end{array}$ & $\begin{array}{c}\text { Recurrence }(-) \\
\quad(n=59)\end{array}$ & $p$ Value \\
\hline Pathological classification & & & & 0.821 \\
\hline Diffuse large B-cell & $74(85.1 \%)$ & $24(32.4 \%)$ & $50(67.6 \%)$ & \\
\hline Others * & $13(14.9 \%)$ & $4(30.8 \%)$ & $9(69.2 \%)$ & \\
\hline Molecular subgroup & & & & 0.672 \\
\hline $\mathrm{GCB}$ & $32(36.8 \%)$ & $12(37.5 \%)$ & $20(62.5 \%)$ & \\
\hline $\mathrm{ABC}$ & $55(63.2 \%)$ & $16(29.1 \%)$ & $39(70.9 \%)$ & \\
\hline Bcl-2 & & & & 0.188 \\
\hline Positive & $66(75.9 \%)$ & $23(34.8 \%)$ & $43(65.2 \%)$ & \\
\hline Negative & $21(24.1 \%)$ & $5(23.8 \%)$ & $16(76.2 \%)$ & \\
\hline Bcl-6 & & & & 0.078 \\
\hline Positive & $69(79.3 \%)$ & $25(36.2 \%)$ & $41(59.4 \%)$ & \\
\hline Negative & $18(20.7 \%)$ & $3(16.7 \%)$ & $15(83.3 \%)$ & \\
\hline p53 & & & & 0.040 \\
\hline Positive & $38(43.7 \%)$ & $18(47.4 \%)$ & $20(52.6 \%)$ & \\
\hline Negative & $49(56.3 \%)$ & $10(20.4 \%)$ & $39(79.6 \%)$ & \\
\hline MUM1 & & & & 0.009 \\
\hline Positive & $50(57.5 \%)$ & $25(50.0 \%)$ & $25(50.0 \%)$ & \\
\hline Negative & $37(42.5 \%)$ & $3(8.1 \%)$ & $34(91.9 \%)$ & \\
\hline c-Мyc & & & & 0.027 \\
\hline Positive & $41(47.1 \%)$ & $19(46.3 \%)$ & $22(53.7 \%)$ & \\
\hline Negative & $46(52.9 \%)$ & $9(19.6 \%)$ & $37(80.4 \%)$ & \\
\hline EBV in situ hybridization & & & & 0.804 \\
\hline Positive & $19(21.8 \%)$ & $6(31.6 \%)$ & $13(68.4 \%)$ & \\
\hline Negative & $68(78.2 \%)$ & $22(32.4 \%)$ & $46(67.6 \%)$ & \\
\hline Ki67 & & & & 0.226 \\
\hline$\geq 50 \%$ & $56(64.4 \%)$ & $21(37.5 \%)$ & $35(62.5 \%)$ & \\
\hline$<50 \%$ & $31(35.6 \%)$ & $7(22.6 \%)$ & $24(77.4 \%)$ & \\
\hline
\end{tabular}

Abbreviations: ABC, activated B-cell-like lymphoma; BCL-2, B-cell lymphoma-2; BCL-6, B-cell lymphoma-6; EBV, Epstein-Barr Virus; GCB, Germinal center B-cell-like lymphoma; MUM1, multiple myeloma-1. * Others mean 10 ALK-negative anaplastic large cell lymphomas, 2 peripheral T-cell lymphomas, and 1 marginal T-cell lymphoma. 


\subsection{Follow-Up and Treatment Response}

All patients completed the five cycles of HDMTX, and the mean follow-up duration was 60.2 months (range, 6-150 months). After induction therapy with HDMTX-based combination chemotherapy, 68 (78.2\%) patients achieved CR and 18 (20.7\%) patients achieved PR; the objective response rate was $98.9 \%$, and only one patient had SD. Therefore, the 68 patients without remnant tumors underwent WBRT at a dose of $36 \mathrm{~Gy}$, and the remaining 19 with remnant tumors underwent whole brain radiotherapy at a dose of $45 \mathrm{~Gy}$. At 2 weeks after the WBRT, none of the remnant tumors were visible on MRI. An additional two cycles of cytarabine chemotherapy were administered to 19 patients who had remnant tumors after induction therapy with the HDMTX-based combination chemotherapy. There were no cases of PD during treatment.

\subsection{PFS and OS}

Twenty-eight (32.2\%) patients experienced recurrence during the follow-up period. The mean time to recurrence was 21.8 months, and the mean PFS was 104.3 months (95\% confidence interval (CI), 90.6-118.0) (Figure 1A). One patient who did not respond to induction treatment with HDMTX experienced recurrence immediately after the end of the cytarabine chemotherapy despite the tumor having disappeared after WBRT; the time to recurrence was 6 months. In the univariate analysis, a normal serum LDH $(p=0.008)$, an ECOG performance score of 2-3 ( $p=0.002)$, lower risk IELSG group $(p<0.001)$, no CSF seeding $(p=0.013)$, homogeneous enhancement on MRI $(p=0.034)$, gross total tumor resection $(p=0.004)$, negative immunoreactivity of BCL2 $(p=0.011)$, negative immunoreactivity of p53 ( $p=0.014)$, negative immunoreactivity of MUM1 $(p=0.002)$, and negative immunoreactivity of c-Myc $(p=0.008)$ were associated with long PFS (Table 4$)$.
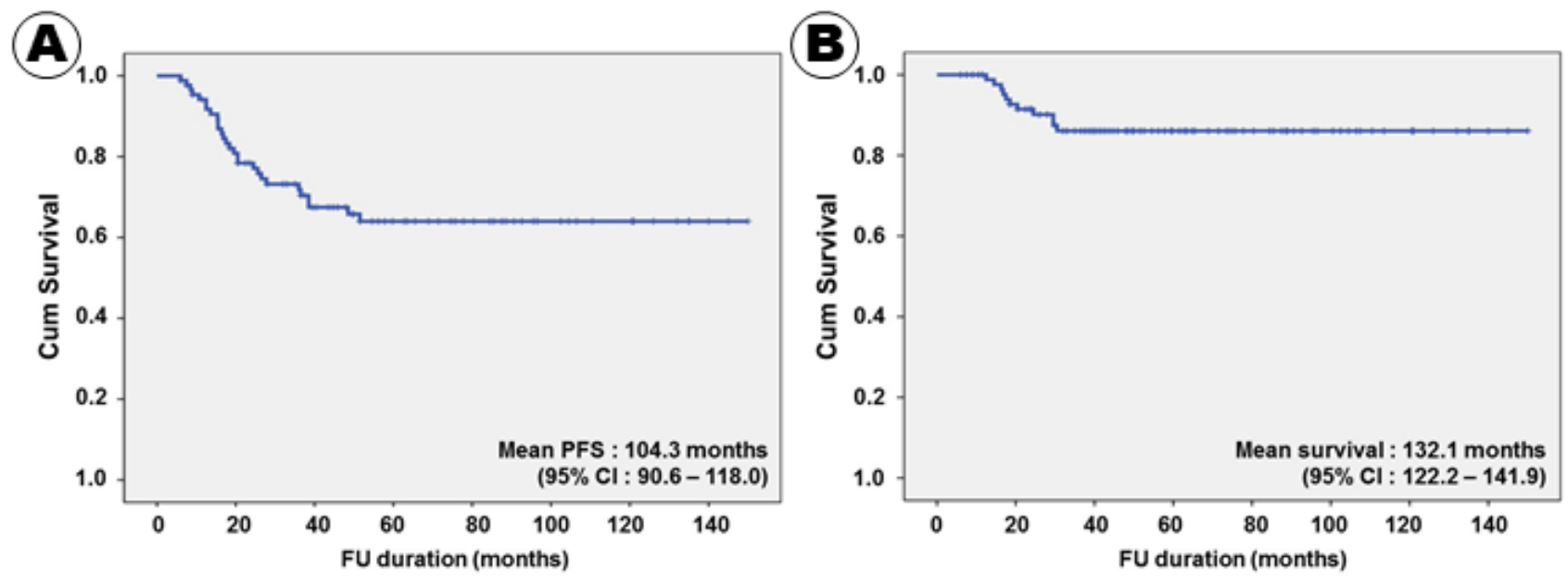

Figure 1. Kaplan-Meier survival curves for progression-free (A) and overall (B) survival.

Eleven (12.6\%) patients succumbed to PCNSL; the mean OS was 132.1 months $(95 \%$ CI, 122.2-141.9) (Figure 1B). The 3- and 5-year survival rates were $92.0 \%$ and $87.4 \%$, respectively. All patients treated with the RTOG 9310 protocol survived for 12 months or more after receiving the PCNSL diagnosis. The shortest survival duration was 12.5 months. In the univariate analysis, a normal serum LDH level $(p=0.002)$, an ECOG performance score of 2-3 ( $p=0.001)$, lower risk IELSG group $(p=0.001)$, unifocal tumor pattern $(p=0.017)$, no CSF seeding $(p=0.005)$, homogeneous enhancement on MRI $(p=0.004)$, gross total tumor resection $(p=0.003)$, negative immunoreactivity of BCL2 $(p<0.001)$, negative immunoreactivity of p53 $(p=0.003)$, negative immunoreactivity of MUM1 $(p=0.001)$, and negative immunoreactivity of c-Myc $(p<0.001)$ were associated with long PFS (Table 4$)$. 
Table 4. Univariate analysis for predicting factors of progression-free and overall survival.

\begin{tabular}{|c|c|c|c|c|c|c|}
\hline Factors & Mean PFS (month) & $\operatorname{HR}(95 \% \mathrm{CI})$ & $p$ Value & $\begin{array}{c}\text { Mean } \\
\text { OS (month) }\end{array}$ & HR $(95 \%$ CI) & $p$ Value \\
\hline Age $<60$ years & $122.5( \pm 7.7)$ & $2.33(0.82-3.74)$ & 0.109 & $127.9( \pm 7.1)$ & $2.05(0.78-3.32)$ & 0.309 \\
\hline$\geq 60$ years & $66.5( \pm 6.5)$ & & & $104.9( \pm 4.5)$ & & \\
\hline Gender Male & $106.1( \pm 9.5)$ & $1.98(0.28-3.68)$ & 0.988 & $127.6( \pm 7.6)$ & $1.97(0.75-3.19)$ & 0.390 \\
\hline Female & $99.4( \pm 8.7)$ & & & $132.2( \pm 6.1)$ & & \\
\hline Ocular involve (+) & $105.3( \pm 6.8)$ & $1.87(0.71-3.03)$ & 0.172 & $134.4( \pm 4.9)$ & $1.55(0.64-2.46)$ & 0.146 \\
\hline involve $(-)$ & $42.0( \pm 8.4)$ & & & $41.9( \pm 8.4)$ & & \\
\hline Serum LDH increase (-) & $116.6( \pm 7.1)$ & $6.18(4.00-8.36)$ & 0.008 & $142.6( \pm 4.1)$ & $7.14(4.62-9.66)$ & 0.002 \\
\hline CSF protein increase $(-)$ & $112.2( \pm 10.3)$ & $1.39(0.80-1.97)$ & 0.387 & $137.7( \pm 6.7)$ & $1.88(0.58-3.18)$ & 0.360 \\
\hline increase (+) & $93.3( \pm 8.0)$ & & & $119.9( \pm 6.5)$ & & \\
\hline ECOG score $0-1$ & $118.7( \pm 6.7)$ & $8.50(5.61-11.39)$ & 0.002 & $145.5( \pm 3.7)$ & $9.04(6.27-11.81)$ & 0.001 \\
\hline score $2-3$ & $34.4( \pm 3.4)$ & & & $40.4( \pm 3.9)$ & & \\
\hline IELSG risk Low & $141.9( \pm 5.3)$ & $13.42(8.45-18.39)$ & $<0.001$ & $145.1( \pm 7.7)$ & $9.53(6.15-12.91)$ & 0.001 \\
\hline Intermediate & $89.3( \pm 7.0)$ & $4.33(2.64-6.02)$ & 0.011 & $136.5( \pm 7.4)$ & $6.55(4.09-9.01)$ & 0.006 \\
\hline High & $31.8( \pm 3.3)$ & & & $14.4( \pm 1.9)$ & & \\
\hline Adjuvant ARA-C Yes & $117.0( \pm 2.5)$ & $1.73(0.75-2.71)$ & 0.362 & $126.7( \pm 7.3)$ & $1.69(0.61-2.77)$ & 0.324 \\
\hline No & $101.0( \pm 7.2)$ & & & $133.1( \pm 6.4)$ & & \\
\hline Multifocal & $68.3( \pm 8.3)$ & & & $73.4( \pm 8.3)$ & & \\
\hline Deep location involve $(-)$ & $109.9( \pm 8.5)$ & $1.52(0.69-2.35)$ & 0.264 & $138.7( \pm 5.4)$ & $1.48(0.51-2.45)$ & 0.126 \\
\hline$(+)$ & $90.0( \pm 9.1)$ & & & $111.2( \pm 8.0)$ & & \\
\hline CSF seeding $(-)$ & $106.0( \pm 6.7)$ & $4.43(1.58-7.28)$ & 0.013 & $135.0( \pm 4.7)$ & $5.74(3.61-7.87)$ & 0.005 \\
\hline$(+)$ & $14.4( \pm 1.9)$ & & & $14.4( \pm 1.9)$ & & \\
\hline Tumor size $<3 \mathrm{~cm}$ & $121.1( \pm 7.8)$ & $1.92(0.94-2.89)$ & 0.089 & $132.2( \pm 6.7)$ & $1.08(0.22-1.94)$ & 0.980 \\
\hline$\geq 3 \mathrm{~cm}$ & $82.2( \pm 9.7)$ & & & $127.4( \pm 7.3)$ & & \\
\hline Peritumoral edema $<2 \mathrm{~cm}$ & $101.6( \pm 6.9)$ & $1.30(0.62-1.97)$ & 0.401 & $120.9( \pm 5.4)$ & $1.52(0.60-2.44)$ & 0.594 \\
\hline$\geq 2 \mathrm{~cm}$ & $95.7( \pm 11.0)$ & & & $129.4( \pm 8.5)$ & & \\
\hline Enhance Homogenous & $130.3( \pm 10.3)$ & $3.35(1.27-5.44)$ & 0.034 & $140.2( \pm 5.4)$ & $6.92(4.05-9.79)$ & 0.004 \\
\hline Heterogeneous & $91.0( \pm 7.6)$ & & & $14.4( \pm 1.9)$ & & \\
\hline Extent of surgery GTR & $128.6( \pm 8.2)$ & $7.12(4.96-9.28)$ & 0.004 & $142.3( \pm 6.1)$ & $7.13(4.68-9.58)$ & 0.003 \\
\hline $\mathrm{Bx}$ & $80.5( \pm 7.4)$ & & & $14.4( \pm 1.9)$ & & \\
\hline Pathology DLBCL & $103.9( \pm 7.3)$ & $1.10(0.43-1.77)$ & 0.919 & $132.8( \pm 5.3)$ & $1.21(0.39-2.03)$ & 0.720 \\
\hline Others & $92.6( \pm 11.4)$ & & & $103.7( \pm 11.1)$ & & \\
\hline
\end{tabular}


Table 4. Cont.

\begin{tabular}{|c|c|c|c|c|c|c|}
\hline Factors & Mean PFS (month) & HR $(95 \%$ CI) & $p$ Value & $\begin{array}{c}\text { Mean } \\
\text { OS (month) }\end{array}$ & HR $(95 \%$ CI) & $p$ Value \\
\hline Cell type Non-GCL & $109.9( \pm 8.2)$ & $1.57(0.61-2.53)$ & 0.304 & $134.8( \pm 5.8)$ & $1.48(0.63-2.33)$ & 0.407 \\
\hline GCL & $84.8( \pm 8.7)$ & & & $107.7( \pm 7.5)$ & & \\
\hline Positive & $92.9( \pm 8.8)$ & & & $14.4( \pm 1.9)$ & & \\
\hline BCL-6 Negative & $128.1( \pm 9.9)$ & $1.83(0.87-2.79)$ & 0.102 & $135.0( \pm 4.7)$ & $2.33(0.97-3.69)$ & 0.084 \\
\hline Positive & $91.4( \pm 6.7)$ & & & $40.4( \pm 5.6)$ & & \\
\hline p53 Negative & $124.5( \pm 6.9)$ & $5.23(2.21-8.24)$ & 0.014 & $135.0( \pm 4.7)$ & 7.50 (3.99-11.01) & 0.003 \\
\hline MUM1 Negative & $140.9( \pm 4.8)$ & $8.21(4.92-11.51)$ & 0.002 & $139.2( \pm 5.1)$ & $10.22(7.45-12.99)$ & 0.001 \\
\hline Positive & $59.1( \pm 5.5)$ & & & $20.8( \pm 5.9)$ & & \\
\hline c-Myc Negative & $124.5( \pm 7.3)$ & $6.09(2.38-9.81)$ & 0.008 & $145.0( \pm 4.7)$ & $14.51(8.77-20.25)$ & $<0.001$ \\
\hline Positive & $64.8( \pm 7.6)$ & & & $14.4( \pm 2.6)$ & & \\
\hline EBV Negative & $103.7( \pm 7.6)$ & $1.23(0.29-2.17)$ & 0.871 & $133.1( \pm 5.6)$ & $1.36(0.55-2.17)$ & 0.728 \\
\hline Positive & $100.1( \pm 12.9)$ & & & $120.5( \pm 10.3)$ & & \\
\hline $\mathrm{Ki} 67<50 \%$ & $120.4( \pm 9.6)$ & $1.95(0.96-2.93)$ & 0.091 & $145.5( \pm 4.4)$ & $1.54(0.86-2.23)$ & 0.246 \\
\hline$\geq 50 \%$ & $81.6( \pm 6.3)$ & & & $101.0( \pm 5.7)$ & & \\
\hline
\end{tabular}

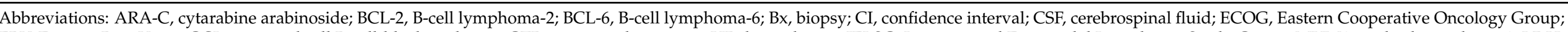

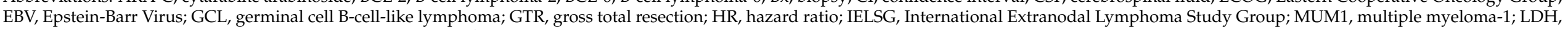
lactate dehydrogenase; OS, overall survival; PFS, progression-free survival. 


\subsection{Multivariate Analysis for Predicting Factors of PFS and OS}

In terms of PFS, 10 factors, namely serum LDH level; ECOG performance score; IELSG risk group; CSF seeding; enhancement pattern on MRI; surgical extent; immunoreactivity of BCL2, p53, MUM1, and c-Myc were positively associated with PFS in the univariate analysis. Six factors, including patient age, ocular involvement, tumor pattern, tumor size, BCL6 immunoreactivity, and Ki67 proliferative index, which tended to be associated with PFS, were included in the multivariate analysis. In conclusion, the following 11 factors were independently associated with PFS: serum LDH level; ECOG performance score; IELSG risk group; CSF seeding; enhancement pattern on MRI; extent of resection; BCL2 expression; BCL6 expression; p53 expression; MUM1 expression; c-Myc expression (Table 5). A KaplanMeier survival curve analysis for PFS, according to various factors, showed the same results (Figure 2).

Table 5. Multivariate analysis for predicting factors of progression-free and overall survival.

\begin{tabular}{|c|c|c|c|c|}
\hline \multirow{2}{*}{ Factors } & \multicolumn{2}{|c|}{ Progression-Free Survival } & \multicolumn{2}{|c|}{ Overall Survival } \\
\hline & HR $(95 \%$ CI $)$ & $p$ Value & HR $(95 \%$ CI $)$ & $p$ Value \\
\hline Age ( $<60$ yrs vs. $\geq 60$ yrs $)$ & $2.23(0.96-3.49)$ & 0.098 & $1.89(0.86-2.92)$ & 0.209 \\
\hline Ocular involvement (No vs. Yes) & $2.07(0.92-3.21)$ & 0.123 & $2.35(0.95-3.75)$ & 0.084 \\
\hline Elevation of LDH (No vs. Yes) & $4.32(1.78-6.86)$ & 0.017 & $2.76(1.27-4.25)$ & 0.043 \\
\hline ECOG score (0-1 vs. 2-3) & $5.18(2.31-8.04)$ & 0.008 & $4.26(2.45-6.07)$ & 0.011 \\
\hline IELSG risk (Low vs. High) & $7.64(4.26-11.02)$ & $<0.001$ & $8.42(5.09-11.76)$ & $<0.001$ \\
\hline (Intermediate vs. High) & $7.05(4.19-9.91)$ & 0.002 & $8.13(4.28-11.98)$ & 0.002 \\
\hline Patterns (Unifocal vs. Multifocal) & $1.94(0.76-3.12)$ & 0.184 & $2.47(0.99-3.94)$ & 0.053 \\
\hline Deep structure involvement (No vs. Yes) & N.A. & & $1.92(0.97-2.87)$ & 0.126 \\
\hline CSF seeding & $4.85(2.54-7.16)$ & 0.002 & $5.22(3.14-7.29)$ & 0.006 \\
\hline Tumor size $(<3 \mathrm{~cm}$ vs. $\geq 3 \mathrm{~cm})$ & $2.44(0.98-3.89)$ & 0.056 & N.A. & \\
\hline $\begin{array}{c}\text { Enhancement } \\
\text { (Homogenous vs. Heterogeneous) }\end{array}$ & $2.73(1.12-3.87)$ & 0.047 & $2.23(0.88-3.48)$ & 0.091 \\
\hline Extent of resection (GTR vs. Bx) & $3.04(1.28-4.79)$ & 0.036 & $2.69(1.14-4.24)$ & 0.048 \\
\hline BCL-2 (Negative vs. Positive) & $2.95(1.33-4.57)$ & 0.042 & $1.74(0.81-2.65)$ & 0.164 \\
\hline BCL-6 (Negative vs. Positive) & $2.88(1.29-4.47)$ & 0.045 & $1.58(0.84-2.31)$ & 0.229 \\
\hline p53 (Negative vs. Positive) & $3.21(1.18-5.24)$ & 0.031 & $3.37(1.66-5.08)$ & 0.036 \\
\hline MUM-1 (Negative vs. Positive) & $3.94(2.17-5.71)$ & 0.028 & $4.62(2.51-6.73)$ & 0.017 \\
\hline c-Myc (Negative vs. Positive) & $3.51(2.33-4.69)$ & 0.035 & $4.08(2.16-6.01)$ & 0.024 \\
\hline Ki67 (<50\% vs. $\geq 50 \%)$ & $2.11(0.88-3.32)$ & 0.122 & $2.31(0.85-3.77)$ & 0.086 \\
\hline
\end{tabular}

Abbreviations: BCL-2, B-cell lymphoma-2; BCL-6, B-cell lymphoma-6; Bx, biopsy; CI, confidence interval; CSF, cerebrospinal fluid; ECOG, Eastern Cooperative Oncology Group; GTR, gross total resection; HR, hazard ratio; IELSG, International Extranodal Lymphoma Study Group; MUM1, multiple myeloma-1; LDH, lactate dehydrogenase; N.A., not assessed.

In terms of OS, 11 factors, namely serum LDH level; ECOG performance score; IELSG risk group; tumor pattern; CSF seeding; enhancement pattern on MRI; surgical extent; BCL2, p53, MUM1, and c-Myc immunoreactivity were positively associated with PFS in the univariate analysis. Three factors, including ocular involvement, deep brain involvement, and BCL6 immunoreactivity, which tended to be associated with PFS, were included in the multivariate analysis. Additionally, patient age and Ki67 proliferative index were included in the multivariate analysis because they are known to be associated with the clinical outcome of PCNSL patients, while evidence for Ki67 can be found in the literature. The following eight factors were independently associated with OS: serum LDH level, ECOG performance score, IELSG risk group, CSF seeding, resection extent, p53 expression, MUM1 expression, and c-Myc expression (Table 5). These eight factors were also independently associated with PFS. A Kaplan-Meier survival curve analysis for OS according to various factors showed the same results (Figure 3). 

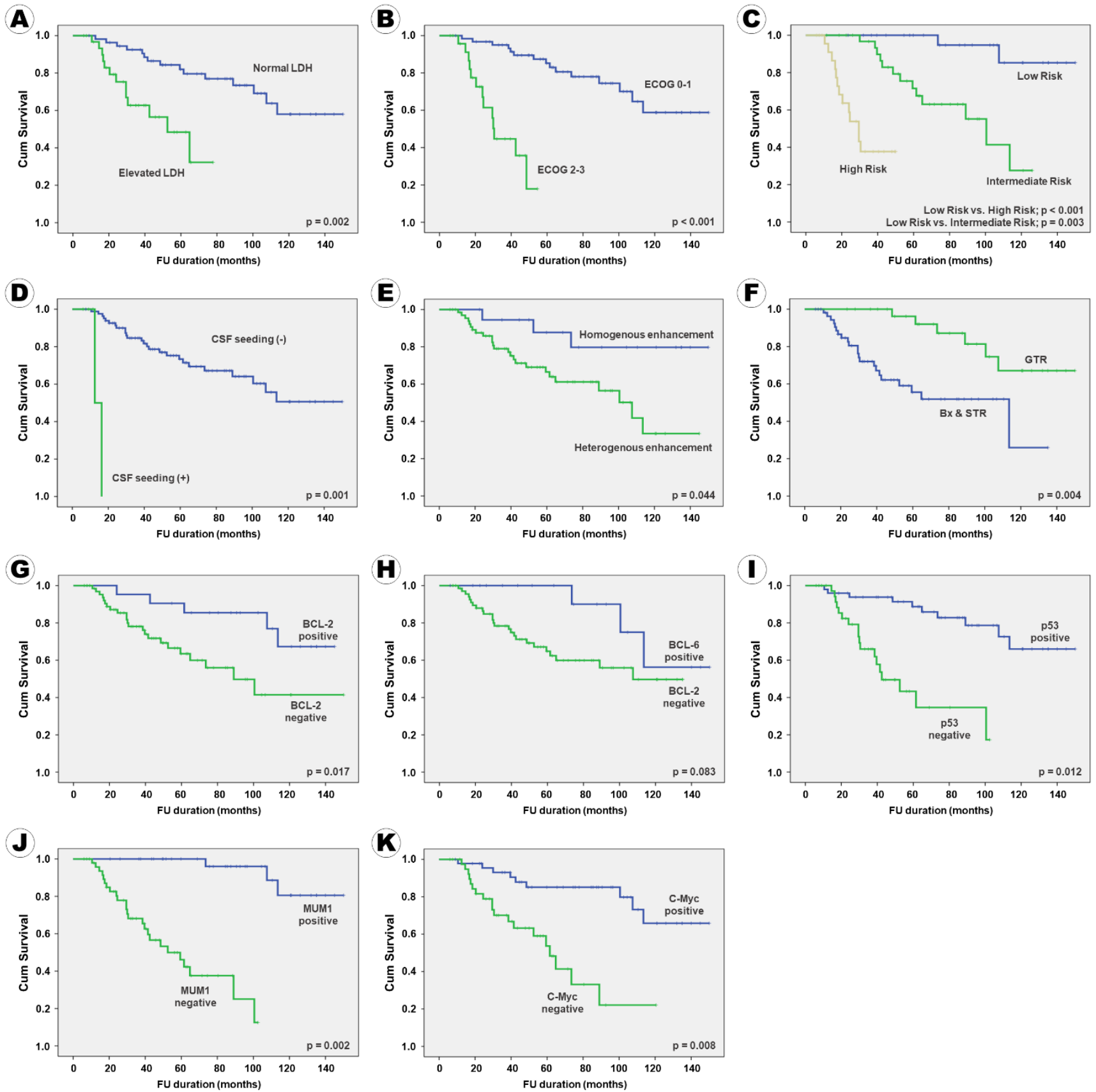

Figure 2. Kaplan-Meier survival curves for progression-free survival according to the clinical, radiological, and pathological factors: (A) serum LDH level, (B) ECOG performance score, (C) IELSG risk group, (D) CSF seeding, (E) enhancement pattern, (F) surgical extent, (G) BCL2 expression, (H) BCL6 expression, (I) p53 expression, (J) MUM1 expression, and (K) c-Myc expression. 

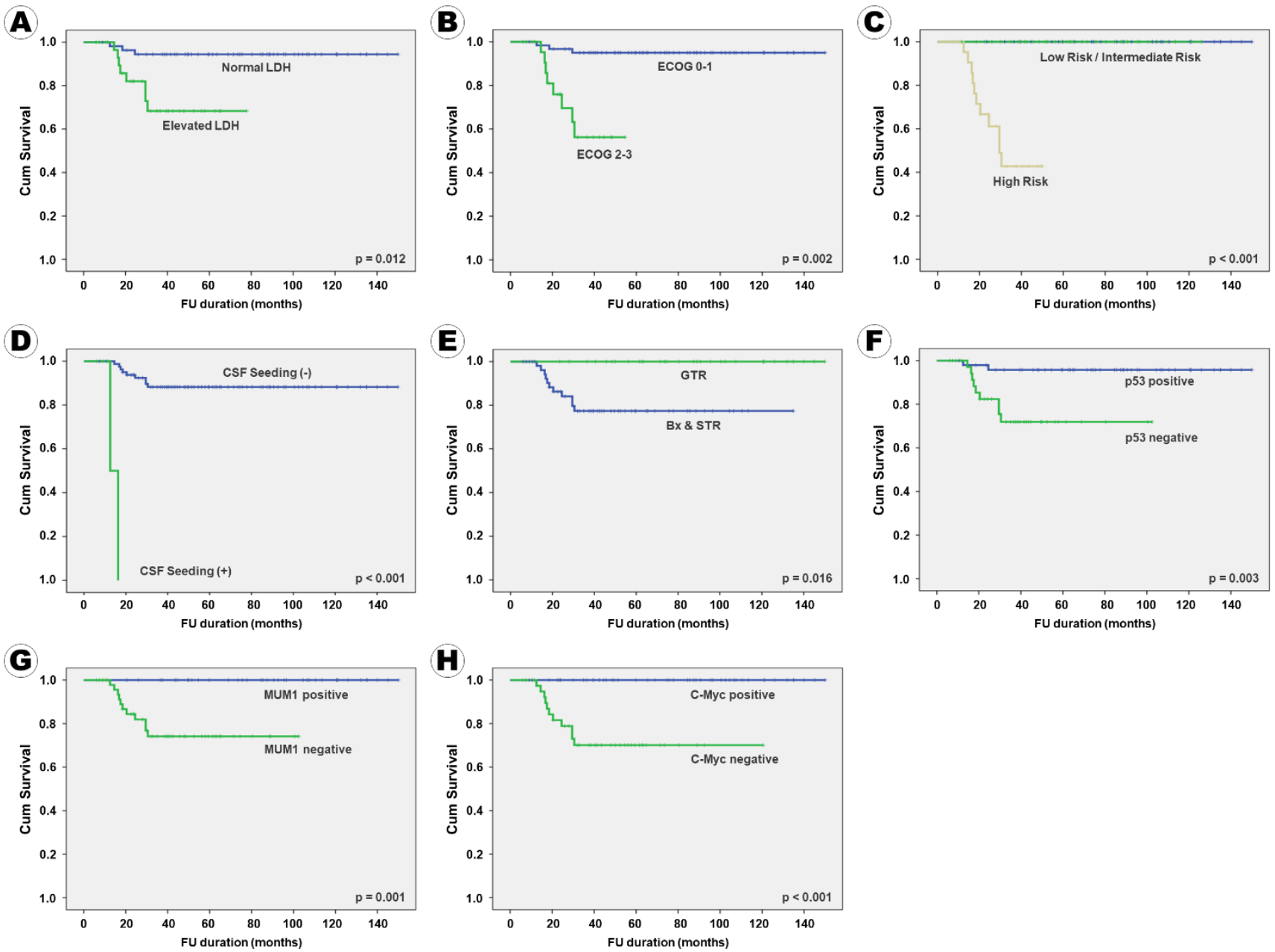

Figure 3. Kaplan-Meier survival curves of overall survival according to the clinical, radiological, and pathological factors: (A) serum LDH level, (B) ECOG performance score, (C) IELSG risk group, (D) CSF seeding, (E) surgical extent, (F) p53 expression, (G) MUM1 expression, and (H) c-Myc expression.

\subsection{Adverse Effects of RTOG 9310 Protocol for PCNSL Patients}

A total of 87 patients completed the five-cycle HDMTX-based combination chemotherapy; among them, 101 adverse effects occurred in 38 patients during the 435 treatment cycles (Supplementary Table S2). The most common side effects were hematologic disturbances such as thrombocytopenia and leukopenia. Among these 101 adverse effects, 100 $(99.0 \%)$ were tolerable at grades 1 and 2 . Only one patient had a life-threatening adverse effect (grade 4 acute renal failure). As renal function recovered after hemodialysis and medical treatment, the two remaining cycles were successfully completed. Fifteen grade 2 adverse effects occurred in eight patients, who continued treatment with a dose reduction of HDMTX.

\section{Discussion}

As the present study was retrospective and performed at a single institute, the value of these results cannot be considered highly significant. However, a relatively large number of patients were treated with a homogeneously consistent protocol, and excellent outcomes were reported compared with previous data from the original RTOG report. Although the clinical results were somewhat different, the treatment methods had many similarities.

In terms of common points between the two studies, the treatment protocol was based on HDMTX plus procarbazine and vincristine. The regimen dosages and number of cycles were identical. In addition, the objective response rate after induction chemotherapy 
using HDMTX-based combination chemotherapy was similar between the two reports (98.9\% in our report and $94 \%$ in the original RTOG report) [12]. However, there were several important differences in the clinical outcomes between the two studies. First, the OS was much longer in our study (mean, 132.1 months; median not reached) than that of the original RTOG report (median, 36.9 months). Additionally, the 3- and 5-year survival rates were much higher in our study versus the RTOG study $(92.0 \%$ vs $52 \%$ and $87.4 \%$ vs $32 \%$, respectively) [12]. Second, PFS was also much longer in our study than the RTOG study (mean, 104.3 months; median not reached vs median, 24.0 months, respectively) in the original RTOG study. In addition, the 3- and 5-year PFS rates were significantly higher in our study versus the original RTOG study $(70.4 \%$ vs $41 \%$ and $63.5 \%$ vs $25 \%$, respectively) [12]. Third, the original RTOG study reported that 53\% of patients experienced grade 3 or 4 toxicity during induction treatment using HDMTX-based combination chemotherapy [12] versus only one patient $(1.1 \%)$ in our study.

Several reasons can be considered for the explanation of different outcomes between our report and the original RTOG report. First, our study had an extremely low rate of adverse effects compared with the original RTOG study (53\% vs $1.1 \%$ cases of grade 3 or 4 toxicity, respectively). HDMTX is known to be highly toxic to the kidneys and nervous system [12,21]. To reduce the toxicity of HDMTX, the RTOG protocol recommends that methotrexate $2.5 \mathrm{~g} / \mathrm{m}^{2}$ be diluted into $500 \mathrm{~mL}$ of D5W solution plus $25 \mathrm{mEq} \mathrm{NaHCO}_{3}$ and infused over $2 \mathrm{~h}$, while leucovorin $20 \mathrm{mg}$ be administered parenterally every $6 \mathrm{~h}$ for 12 doses starting $24 \mathrm{~h}$ after the systemic HDMTX infusion starts. Leucovorin rescue is important for reducing the adverse effects of HDMTX, and its dose titration should be determined according to the MTX serum level. However, it was practically impossible for our institute to receive a laboratory report of serum MTX levels within $48 \mathrm{~h}$ after blood sampling due to our laboratory system. As we could not deliver the optimal leucovorin rescue, the strict alkalinization of urine was targeted with an intravenous $\mathrm{NaHCO}_{3}$ infusion. After the occurrence of grade 4 toxicity due to inappropriate leucovorin rescue in the early period of PCNSL treatment at our institute using RTOG protocol 9310, we began to control the urine $\mathrm{pH}$ at over 7.0 using the dynamic dose of $\mathrm{NaHCO}_{3}$ infusion mixed in $\mathrm{D} 5 \mathrm{~W}$ solution. Thereafter, there were no cases of grade 3-4 toxicity during induction chemotherapy using HDMTX. Without serious treatment-induced toxicity, most patients could finish the five cycles of HDMTX-based combination chemotherapy.

Second, the modification radiotherapy doses and fractionations differed between the studies. The initial RTOG report planned WBRT for a total dose of $45 \mathrm{~Gy}$ in 1.8-Gy fractions. Approximately halfway through the original RTOG study, evidence was growing from the single-institution experience that long-term survivors of combined HDMTXbased combined chemotherapy and cranial irradiation were developing permanent severe neurotoxicity [13]. The study was then modified so that those patients who achieved a CR at the end of the five cycles of HDMTX-based combination chemotherapy would receive a hyperfractionated WBRT course for a total dose of 36 Gy given in 1.20-Gy fractions twicedaily for 15 days; the twice-daily RT doses were separated by a minimum of $6 \mathrm{~h}$. However, all patients in our study underwent WBRT with a reduced dose of 36 Gy in 1.8-Gy fractions for 20 days when no remnant tumor was detected after induction chemotherapy with HDMTX-based combination chemotherapy. A relatively large number of patients (78.2\%) underwent WBRT with a reduced dose of $36 \mathrm{~Gy}$ in 1.8-Gy fractions for 20 days compared with the original RTOG report. Although our study did not show any data on the decline of cognitive function after treatment due to its short follow-up duration, modification of the WBRT according to the presence of remnant tumor could reduce the adverse effects of therapy.

Third, consolidation chemotherapy using cytarabine arabinoside was not used to treat patients who had no remnant tumor after induction chemotherapy and WBRT. The original RTOG protocol recommends that all patients receive two courses of high-dose cytarabine at the completion of cranial irradiation. Each course includes two doses of cytarabine $3 \mathrm{~g} / \mathrm{m}^{2} /$ day infused over $3 \mathrm{~h}$ and administered $24 \mathrm{~h}$ apart. All patients in the original 
RTOG study were treated with high-dose cytarabine chemotherapy, while only 19 (21.8\%) received high-dose cytarabine chemotherapy in our study because tumors remained after induction chemotherapy and WBRT. However, there was no difference in recurrence rate between patients who were and those who were not treated with high-dose cytarabine chemotherapy, which suggested that it is possible for patients without remnant tumor after induction chemotherapy to not be treated with consolidation chemotherapy with high-dose cytarabine.

The present study highlights the importance of a good patient condition during treatment as a major factor for predicting clinical outcomes. Our minimal modification of the RTOG protocol, such as maintaining a urine $\mathrm{pH}$ over 7.0, during HDMTX treatment, the application of WBRT dose and fraction according to remnant tumor status after induction chemotherapy, and the omission of consolidation chemotherapy with high-dose cytarabine, could reduce the adverse effects. As a result, patients successfully completed the full treatment cycles, which improved their clinical outcomes. In fact, our study showed that good patients' performance status estimated by the ECOG performance score, which is commonly applied to other cancer patients, is a strong prognostic factor.

Although the original RTOG report did not show any pathological information associated with clinical outcomes, several studies have suggested that certain biomarkers are associated with prognosis. Among them, BCL2 and BCL6 have been studied worldwide for their prognostic role in PCNSL [17,22-24]. Although our study findings suggested that immunohistochemical positivity for BCL2 and BCL6 should be independently associated with a short PFS rather than OS in a multivariate analysis, controversy persists regarding the role of BCL2 and BCL6 protein expression on the prognosis of PCNSL patients. It is unclear whether the inconclusive findings across BCL2 studies were caused by different cutoffs used to define its positivity, which ranged from $50 \%$ to $70 \%$ [25-28]. However, Tapia et al. evaluated several cutoffs to define BCL2 positivity $(30 \%, 50 \%$, and $70 \%)$ and found no significant association between BCL2 expression and PCNSL patient prognosis [29]. Therefore, further comprehensive studies are essential to determine the prognostic role of BCL2 and BCL6 in PCNSL patients.

In addition, c-Myc is a widely studied biomarker in PCNSL with DLBCL cells. The expression of c-Myc proteins has been described in up to 70-90\% of PCNSL DLBCL cases $[30,31]$. Although systemic DLBCL with high c-Myc expression has been associated with a worse prognosis, the findings were inconclusive for PCNSL with DLBCL cells [21]. Interestingly, c-Myc gene rearrangements occur at significantly lower frequencies $(3-8 \%)$ [30,32], which suggests that increased c-Myc protein expression might be attributable to other genetic aberrations, such as MYC gene mutations or altered regulation of expression (e.g., through epigenetic modifications) independent of $M Y C$ gene rearrangements [21]. Although our study findings suggested that a high expression of c-Myc is strongly associated with PFS and OS with a cutoff value of $60 \%$, there are no conclusive findings regarding the prognostic role of c-Myc in PCNSL patients. Several studies have shown that c-Myc protein overexpression (defined as immunohistochemical staining with at least $40 \%$ tumor cells) was associated with worse OS and PFS [26,27,33], but other studies found no significant difference in prognosis [25,30]. It is important to note that these studies used the same cutoff to define positive c-Myc protein expression. Our cutoff value of c-Myc expression in immunohistochemical staining was 60\%; Tapia et al. used a cutoff value of $30 \%$, which was relatively low compared with ours, to define c-Myc positivity and reported an association between c-Myc expression and poor outcome [29]. It remains unclear why these results have varied across studies, and it does not seem to be related to the c-Myc antibodies used.

With minimal modification of the original RTOG 9310 protocol for treating PCNSL, our study showed much improved clinical outcomes compared with the original RTOG report and suggested that several pathological biomarkers were associated with the prognosis of PCNSL patients treated with the RTOG 9310 protocol. However, several limitations must be noted. First, inherent bias was introduced by the retrospective nature of the study. 
We attempted to reduce this bias by collecting patient data from complete medical and radiological records and recruiting patients treated using the single and consistent RTOG 9310 protocol. Although multiple investigators without patient information independently reviewed the pathological slides and radiological images, we cannot clearly claim that no bias originated from this retrospective study. Despite these efforts, however, the conclusions drawn here require further validation through prospective and randomized clinical trials.

Second, although two different investigators examined and recorded the immunohistochemical staining data in the pathological report of the samples, it is uncertain whether the immunohistochemical results obtained in the pathological reports were correct because the assessment of immunohistochemical staining results is qualitative and often subjective. Reasonable run-to-run reproducibility with repeated reviews of pathological slides is essential for the proper implementation of these cutoff levels. In addition, threshold levels require adjustments according to the sensitivity of the method used. For this reason, we used specificity-sensitivity testing to determine the optimal cutoff level. However, additional studies are necessary to validate the reproducibility of our immunohistochemical staining method.

Third, our study used the traditional treatment protocol, which has not been updated for 13 years. New treatment strategies, such as combination therapy with rituximab and temozolomide, have recently evolved to achieve a median OS of 90 months [21]. Although several clinical trials are also investigating the role of induction rituximab in newly diagnosed PCNS DLBCL randomized to rituximab, MTX, teniposide, Bis-chlorethylnitrosourea (BCNU), prednisolone (R-MBVP), or MBVP alone [24], limited PCNSL patients are treated with new regimens rather than MTX in Korea because it lacks National Health Insurance approval. Therefore, combination treatment based on HDMTX is still generally considered the first-line treatment for PCNSL patients in Korea. However, with the accumulation of experience treating PCNSL patients with HDMTX, clinical outcomes have improved to as long as an OS of 131.1 months, while the adverse effects have also significantly reduced.

Finally, our study did not show the results of molecular genetic analyses, which has been updated and proven to have a prognostic role in PCNSL patients. For example, classic mutation profiles including MYD88 L265P and CD79B driving oncogenic toll-like receptor signaling, BCL2 and $M Y C$ rearrangements in nodal/systemic activated B-cell-like lymphoma-type DLBCL, nuclear factor kappa- $\beta$ signaling pathway dysregulation, and copy number variations have been shown to play an oncogenic function in PCNSL [34]. Comprehensive genetic and epigenetic research is required to prove the role of several molecular markers in predicting the prognosis of patients with PCNSL.

\section{Conclusions}

The present study investigated the PFS and OS of PCNSL patients who were treated with the RTOG 9310 protocol, which consists of HDMTX-based combination chemotherapy followed by WBRT. Despite the traditional protocol using HDMTX as the standard, the clinical outcomes were much better than those of other reports of recently updated treatment regimens. The minimal modification of the protocol through our accumulated experience of HDMTX-based combination chemotherapy reduced the serious adverse effects, which helped patients complete the full cycles of induction chemotherapy and consolidation radiotherapy. As a result, successful treatment achieved better outcomes in patients with PCNSL. However, further prospective and randomized clinical trials are necessary to validate our results.

Supplementary Materials: The following are available online at https: / www.mdpi.com/article / 10.3390 / curroncol28060393/s1, Table S1: Treatment flow chart of RTOG 9310 protocol for primary central nervous system lymphoma, Table S2: Common Terminology Criteria for Adverse Events in RTOG9310 protocol (total 435 cycles).

Author Contributions: All authors contributed significantly to this article as follows: Conceptualization, Y.Z.K. and S.H.K.; methodology, J.K., T.G.K., and S.H.K.; software, J.K., J.E.P., and M.L.; 
validation, H.W.L.; formal analysis, H.W.L., T.G.K., and S.H.K.; investigation, J.K. and Y.Z.K.; resources, J.K., J.E.P., and M.L.; data curation, J.K. and Y.Z.K.; writing-original draft preparation, J.K. and Y.Z.K.; writing-review and editing, J.K. and Y.Z.K.; visualization, J.K. and Y.Z.K.; supervision, Y.Z.K.; project administration, Y.Z.K.; funding acquisition, Y.Z.K. All authors have read and agreed to the published version of the manuscript.

Funding: This research was supported by a National Research Foundation of Korea grant funded by the Korean Government (Ministry of Science and ICT) (grant no. NRF 2019R 1F1A 1054681). This study was also financially supported by the Sungkyun Research Fund, Sungkyunkwan University (2016) and the Samsung Changwon Hospital Research Fund (2020).

Institutional Review Board Statement: The institutional review board of Sungkyunkwan University Samsung Changwon Hospital approved the study protocol (SCMC 2021-07-007). This study was conducted in accordance with the guidelines of the Declaration of Helsinki for biomedical research. The need for written informed consent was waived owing to the study's retrospective nature.

Informed Consent Statement: The institutional review board of Sungkyunkwan University Samsung Changwon Hospital waived the need for written informed consent owing to the study's retrospective nature.

Data Availability Statement: The data presented in this study are available on request from the corresponding author. The data are not publicly available due to sensitive clinical data involved.

Acknowledgments: We thank Young Min Kim and Mi-Ok Sunwoo (Department of Radiology, Samsung Changwon Hospital, Changwon, Korea) for reviewing the neuroradiological images, Eun Hee Lee for reviewing the pathological images, and Young Wook Kim (Department of Biostatistics, Samsung Changwon Hospital, Changwon, Korea) for assisting with the statistical analysis detailed in this work.

Conflicts of Interest: The authors declare no competing interests concerning the materials or methods used in this study or the findings specified herein. The funders had no role in the study design; data collection, analyses, or interpretation; writing of the manuscript; or decision to publish the results.

\section{References}

1. Hoang-Xuan, K.; Bessell, E.; Bromberg, J.; Hottinger, A.F.; Preusser, M.; Rudà, R.; Schlegel, U.; Siegal, T.; Soussain, C.; Abacioglu, U.; et al. Diagnosis and treatment of primary CNS lymphoma in immunocompetent patients: Guidelines from the European Association for Neuro-Oncology. Lancet Oncol. 2015, 16, e322-e332. [CrossRef]

2. Dho, Y.S.; Jung, K.W.; Ha, J.; Seo, Y.B.; Park, C.K.; Won, Y.J.; Yoo, H. An Updated Nationwide Epidemiology of Primary Brain Tumors in Republic of Korea, 2013. Brain Tumor Res. Treat. 2017, 5, 16-23. [CrossRef]

3. Bessell, E.M.; Dickinson, P.; Dickinson, S.; Salmon, J. Increasing age at diagnosis and worsening renal function in patients with primary central nervous system lymphoma. J. Neurooncol. 2011, 104, 191-193. [CrossRef]

4. Villano, J.L.; Koshy, M.; Shaikh, H.; Dolecek, T.A.; McCarthy, B.J. Age, gender, and racial differences in incidence and survival in primary CNS lymphoma. Br. J. Cancer 2011, 105, 1414-1418. [CrossRef] [PubMed]

5. O'Neill, B.P.; Decker, P.A.; Tieu, C.; Cerhan, J.R. The changing incidence of primary central nervous system lymphoma is driven primarily by the changing incidence in young and middle-aged men and differs from time trends in systemic diffuse large B-cell non-Hodgkin's lymphoma. Am. J. Hematol. 2013, 88, 997-1000. [CrossRef]

6. O'Neill, B.P.; O'Fallon, J.R.; Earle, J.D.; Colgan, J.P.; Brown, L.D.; Krigel, R.L. Primary central nervous system non-Hodgkin's lymphoma: Survival advantages with combined initial therapy? Int. J. Radiat. Oncol. Biol. Phys. 1995, 33, 663-673. [CrossRef]

7. Schultz, C.; Scott, C.; Sherman, W.; Donahue, B.; Fields, J.; Murray, K.; Fisher, B.; Abrams, R.; Meis-Kindblom, J. Pre-irradiation chemotherapy with cyclophosphamide, doxorubicin, vincristine, and dexamethasone (CHOD) for PCNSL: Initial report of Radiation Therapy Oncology Group (RTOG) protocol 88-06. J. Clin. Oncol. 1996, 14, 556-564. [CrossRef] [PubMed]

8. Mead, G.M.; Bleehen, N.M.; Gregor, A.; Bullimore, J.; Shirley, D.; Rampling, R.P.; Trevor, J.; Glaser, M.G.; Lantos, P.; Ironside, J.W.; et al. A Medical Research Council randomized trial in patients with primary cerebral non-Hodgkin lymphoma: Cerebral radiotherapy with and without cyclophosphamide, doxorubicin, vincristine, and prednisone chemotherapy. Cancer 2000, 89 , 1359-1370. [CrossRef]

9. Hiraga, S.; Arita, N.; Ohnishi, T.; Kohmura, E.; Yamamoto, K.; Oku, Y.; Taki, T.; Sato, M.; Aozasa, K.; Yoshimine, T. Rapid infusion of high-dose methotrexate resulting in enhanced penetration into CSF and intensified tumour response in PCNSL. J. Neurosurg. 1999, 91, 221-230. [CrossRef] [PubMed]

10. Glass, J.; Gruber, M.L.; Cher, L.; Hochberg, F.H. Preirradiation methotrexate chemotherapy of primary central nervous system lymphoma: Long-term outcome. J. Neurosurg. 1994, 81, 188-195. [CrossRef]

11. Grommes, C.; DeAngelis, L.M. Primary CNS Lymphoma. J. Clin. Oncol. 2017, 35, 2410-2418. [CrossRef] [PubMed] 
12. DeAngelis, L.M.; Seiferheld, W.; Schold, S.C.; Fisher, B.; Schultz, C.J. Combination chemotherapy and radiotherapy for primary central nervous system lymphoma: Radiation Therapy Oncology Group Study 93-10. J. Clin. Oncol. 2002, 20, 4643-4648. [CrossRef]

13. Fisher, B.; Seiferheld, W.; Schultz, C.; DeAngelis, L.; Nelson, D.; Schold, S.C.; Curran, W.; Mehta, M. Secondary analysis of Radiation Therapy Oncology Group study (RTOG) 9310: An intergroup phase II combined modality treatment of primary central nervous system lymphoma. J. Neuro-Oncol. 2005, 74, 201-205. [CrossRef]

14. Oken, M.M.; Creech, R.H.; Tormey, D.C.; Horton, J.; Davis, T.E.; McFadden, E.T.; Carbone, P.P. Toxicity and response criteria of the Eastern Cooperative Oncology Group. Am. J. Clin. Oncol. 1982, 5, 649-655. [CrossRef]

15. Ferreri, A.J.; Blay, J.Y.; Reni, M.; Pasini, F.; Spina, M.; Ambrosetti, A.; Calderoni, A.; Rossi, A.; Vavassori, V.; Conconi, A.; et al. Prognostic scoring system for primary CNS lymphomas: The International Extranodal Lymphoma Study Group Experience. J. Clin. Oncol. 2003, 21, 266-272. [CrossRef] [PubMed]

16. Arber, D.A.; Orazi, A.; Hasserjian, R.; Thiele, J.; Borowitz, M.J.; Le Beau, M.M.; Bloomfield, C.D.; Cazzola, M.; Vardiman, J.W. The 2016 revision to the World Health Organization classification of myeloid neoplasms and acute leukemia. Blood 2016, 127, 2391-2405. [CrossRef]

17. Lauw, M.I.S.; Lucas, C.H.G.; Ohgami, R.S.; Wen, K.W. Primary Central Nervous System Lymphomas: A diagnostic overview of key histomorphologic, immunophenotypic, and genetic features. Diagnostics 2020, 10, 1076. [CrossRef] [PubMed]

18. Cheson, B.D.; Horning, S.J.; Coiffier, B.; Shipp, M.A.; Fisher, R.I.; Connors, J.M.; Lister, T.A.; Vose, J.; Grillo-López, A.; Hagenbeek, A.; et al. Report of an international workshop to standardize response criteria for non-Hodgkin's lymphomas. NCI Sponsored International Working Group. J. Clin. Oncol. 1999, 17, 1244. [CrossRef]

19. National Institutes of Health and National Cancer Institute. Common Terminology Criteria for Adverse Events (CTCAE) Version 5.0. Available online: https:/ / ctep.cancer.gov/protocolDevelopment/electronic_applications/ctc.htm\#ctc_50 (accessed on 5 July 2021).

20. Eng, J. Receiver operating characteristic analysis: A primer. Acad. Radiol. 2005, 12, 909-916. [CrossRef]

21. Glass, J.; Won, M.H.; Schultz, C.J.; Brat, D.; Bartlett, N.L.; Suh, J.H.; Werner-Wasik, M.; Fisher, B.J.; Liepman, M.K.; Augspurger, M.; et al. Phase I and II study of induction chemotherapy with methotrexate, rituximab, and temozolomide, followed by whole-brain radiotherapy and postirradiation temozolomide for primary CNS lymphoma: NRG Oncology RTOG 0227. J. Clin. Oncol. 2016, 34, 1620-1625. [CrossRef]

22. Nosrati, A.; Monabati, A.; Sadeghipour, A.; Radmanesh, F.; Safaei, A.; Movahedinia, S. MYC, BCL2, and BCL6 rearrangements in primary central nervous system lymphoma of large B cell type. Ann. Hematol. 2019, 98, 169-173. [CrossRef] [PubMed]

23. Kreher, S.; Johrens, K.; Strehlow, F.; Martus, P.; Borowiec, K.; Radke, J.; Heppner, F.; Roth, P.; Thiel, E.; Pietsch, T.; et al. Prognostic impact of B-cell lymphoma 6 in primary CNS lymphoma. Neuro. Oncol. 2015, 17, 1016-1021. [CrossRef]

24. Löw, S.; Han, C.H.; Batchelor, T.T. Primary central nervous system lymphoma. Ther. Adv. Neurol. Disord. 2018, 11, 1-16. [CrossRef]

25. Makino, K.; Nakamura, H.; Shinojima, N.; Kuroda, J.-I.; Yano, S.; Mikami, Y.; Mukasa, A. BCL2 expression is associated with a poor prognosis independent of cellular origin in primary central nervous system diffuse large B-cell lymphoma. J. Neuro-Oncol. 2018, 140, 115-121. [CrossRef] [PubMed]

26. Shi, Q.-Y.; Feng, X.; Bao, W.; Ma, J.; Lv, J.-H.; Wang, X.; Rao, Q.; Shi, Q.-L. MYC/BCL2 co-expression is a stronger prognostic factor compared with the cell-of-origin classification in primary CNS DLBCL. J. Neuropathol. Exp. Neurol. 2017, 76, 942-948. [CrossRef]

27. Kim, S.; Nam, S.J.; Kwon, D.; Kim, H.; Lee, E.; Kim, T.M.; Heo, D.S.; Park, S.H.; Kim, C.W.; Jeon, Y.K. MYC and BCL2 overexpression is associated with a higher class of Memorial Sloan-Kettering Cancer Center prognostic model and poor clinical outcome in primary diffuse large B-cell lymphoma of the central nervous system. BMC. Cancer 2016, 16, 363. [CrossRef]

28. Liu, J.; Wang, Y.; Liu, Y.; Liu, Z.; Cui, Q.; Ji, N.; Sun, S.; Wang, B.; Wang, Y.; Sun, X.; et al. Immunohistochemical profile and prognostic significance in primary central nervous system lymphoma: Analysis of 89 cases. Oncol. Lett. 2017, 14, 5505-5512. [CrossRef] [PubMed]

29. Tapia, G.; Baptista, M.-J.; Muñoz-Marmol, A.-M.; Gaafar, A.; Puente-Pomposo, M.; Garcia, O.; Marginet-Flinch, R.; Sanz, C.; Navarro, J.-T.; Sancho, J.-M.; et al. MYC protein expression is associated with poor prognosis in primary diffuse large B-cell lymphoma of the central nervous system. APMIS 2015, 123, 596-603. [CrossRef] [PubMed]

30. Gill, K.Z.; Iwamoto, F.; Allen, A.; Hoehn, D.; Murty, V.V.; Alobeid, B.; Bhagat, G. MYC Protein Expression in Primary Diffuse Large B-Cell Lymphoma of the Central Nervous System. PLoS ONE 2014, 9, e114398.

31. Cady, F.M.; O’Neill, B.P.; Law, M.E.; Decker, P.A.; Kurtz, D.M.; Giannini, C.; Porter, A.B.; Kurtin, P.J.; Johnston, P.B.; Dogan, A.; et al. Del(6)(q22) and BCL6 Rearrangements in Primary CNS Lymphoma Are Indicators of an Aggressive Clinical Course. J. Clin. Oncol. 2008, 26, 4814-4819. [CrossRef]

32. Brunn, A.; Nagel, I.; Montesinos-Rongen, M.; Klapper, W.; Vater, I.; Paulus, W.; Hans, V.; Blümcke, I.; Weis, J.; Siebert, R.; et al. Frequent triple-hit expression of MYC, BCL2, and BCL6 in primary lymphoma of the central nervous system and absence of a favorable MYC low BCL2 low subgroup may underlie the inferior prognosis as compared to systemic diffuse large B cell lymphomas. Acta Neuropathol. 2013, 126, 603-605. [CrossRef] [PubMed]

33. Son, S.M.; Ha, S.Y.; Yoo, H.Y.; Oh, D.; Kim, S.J.; Kim, W.S.; Ko, Y.H. Prognostic impact of MYC protein expression in central nervous system diffuse large B-cell lymphoma: Comparison with MYC rearrangement and MYC mRNA expression. Mod. Pathol. 2016, 30, 4-14. [CrossRef] [PubMed]

34. King, R.L.; Goodlad, J.R.; Calaminici, M.; Dotlic, S.; Montes-Moreno, S.; Oschlies, I.; Ponzoni, M.; Traverse-Glehen, A.; Ott, G.; Ferry, J.A. Lymphomas arising in immune-privileged sites: Insights into biology, diagnosis, and pathogenesis. Virchows Arch. 2020, 476, 647-665. [CrossRef] [PubMed] 